\title{
(NZ)CH...O Contacts assist crystallization of a ParB-like nuclease
} Neil Shaw ${ }^{1}$, Chongyun Cheng1, Wolfram Tempel ${ }^{2}$, Jessie Chang' ${ }^{2}$, Joseph $\mathrm{Ng}^{3}$, Xin-Yu Wang ${ }^{1}$, Sarah Perrett ${ }^{1}$, John Rose ${ }^{2}$, Zihe Rao ${ }^{1,4}$, Bi-Cheng Wang ${ }^{2}$ and Zhi-Jie Liu*1,2

\author{
Address: ${ }^{1}$ National Laboratory of Biomacromolecules, Institute of Biophysics, Chinese Academy of Sciences, Beijing, 100101, China, ${ }^{2}$ Southeast \\ Collaboratory for Structural Genomics, Department of Biochemistry and Molecular Biology, University of Georgia, GA 30602, USA, ${ }^{3}$ Laboratory \\ of Structural Biology and Department of Biological Sciences, University of Alabama in Huntsville, Huntsville, AL 35899, USA and ${ }^{4}$ Laboratory of \\ Structural Biology, Tsinghua University, 100084, China \\ Email: Neil Shaw - neilshaw@moon.ibp.ac.cn; Chongyun Cheng - ccy@moon.ibp.ac.cn; Wolfram Tempel - tempel@gmail.com; \\ Jessie Chang - Jessie@secsg.uga.edu; Joseph Ng - ngj@email.uah.edu; Xin-Yu Wang - xinyuwang_cn@yahoo.com.cn; \\ Sarah Perrett - sperrett@ibp.ac.cn; John Rose - rose@bcl4.bmb.uga.edu; Zihe Rao - raozh@xtal.tsinghua.edu.cn; Bi- \\ Cheng Wang - wang@bcl1.bmb.uga.edu; Zhi-Jie Liu* - zjliu@ibp.ac.cn \\ * Corresponding author
}

Published: 7 July 2007

BMC Structural Biology 2007, 7:46 doi:10.1 186/1472-6807-7-46
Received: 10 February 2007

Accepted: 7 July 2007

This article is available from: http://www.biomedcentral.com/1472-6807/7/46

(C) 2007 Shaw et al; licensee BioMed Central Ltd.

This is an Open Access article distributed under the terms of the Creative Commons Attribution License (http://creativecommons.org/licenses/by/2.0), which permits unrestricted use, distribution, and reproduction in any medium, provided the original work is properly cited.

\begin{abstract}
Background: The major bottleneck for determination of $3 \mathrm{D}$ structures of proteins using $\mathrm{X}$-rays is the production of diffraction quality crystals. Often proteins are subjected to chemical modification to improve the chances of crystallization

Results: Here, we report the successful crystallization of a nuclease employing a reductive methylation protocol. The key to crystallization was the successful introduction of 44 new cohesive (NZ) $\mathrm{CH}$...O contacts $(3.2-3.7 \AA)$ by the addition of 2 methyl groups to the side chain amine nitrogen (NZ) of 9 lysine residues of the nuclease. The new contacts dramatically altered the crystallization properties of the protein, resulting in crystals that diffracted to $1.2 \AA$ resolution. Analytical ultracentrifugation analysis and thermodynamics results revealed a more compact protein structure with better solvent exclusion of buried $\operatorname{Trp}$ residues in the folded state of the methylated protein, assisting crystallization.
\end{abstract}

Conclusion: In this study, introduction of novel cohesive (NZ)CH...O contacts by reductive methylation resulted in the crystallization of a protein that had previously resisted crystallization in spite of extensive purification and crystallization space screening. Introduction of (NZ)CH... contacts could provide a solution to crystallization problems for a broad range of protein targets.

\section{Background}

The resolution and accuracy of the structural information provided by X-rays is unsurpassed when compared to other techniques employed to resolve the $3 \mathrm{D}$ structure of proteins [1]. However, not all proteins can be made available for X-ray diffraction studies because of the inherent difficulty in obtaining single crystals of adequate size and quality [2]. A subset of such proteins that resist crystallization can be salvaged by employing a reductive methylation protocol [3-11]. A $26 \mathrm{kD}$ nuclease from Pyrococcus furiosus used in the current study had resisted crystallization inspite of extensive purification and crystallization 
space screening. A number of techniques have been developed to improve a given target's potential for crystallization. Truncation of disordered regions [12,13], mutagenesis of surface residues [14-16] and chemical modification of proteins [3] have been proven effective in this regard and a number of protein structures have been solved successfully. We decided to modify the surface lysines of the nuclease by reductive methylation in an attempt to crystallize the protein. The rationale behind targeting the amine nitrogen (NZ) of the nuclease arose from a number of considerations. Lysines harbouring free $\mathrm{NZ}$ atoms almost always reside on the surface of protein molecules [17]. The thermodynamic cost for ordering the highly flexible solvent exposed side chains of lysines is exorbitant [18]. Since crystallization is a surface phenomenon, a disordered lysine side chain has a profound negative impact on the formation of stable, uniform, inter molecular contacts essential for packaging of protein molecules in a crystal lattice. Interestingly, we found that methylation of surface lysines resulted in a decrease in the free energy of folding of the protein. This is consistent with the entropic cost of reduced flexibility in the native state due to formation of new intra molecular interactions, which in turn will lower the barrier to crystallization. We further probed the effect of methylation at the molecular level and show that cohesive (NZ)CH...O bonds assisted crystallization of the nuclease.

\section{Results}

\section{Generation of intra molecular contacts}

After purifying the protein to homogeneity, two methyl groups were covalently linked to the free amine nitrogen of lysine residues [3-11] (Figure 1A) by treating the protein with formaldehyde and dimethylamine borane complex (DMAB). Electron density for only 9 amine nitrogens out of 32 (including the $\mathrm{N}$-terminal amine group), could be unambiguously assigned as dimethylated (Figure 1B).

The methyl carbon is protonated because of the strong electron withdrawing nature of the NZ atom. The protonated methyl carbon can form ionic interactions with carbonyl and carboxyl oxygens of surrounding residues. The (NZ)CH...O bond formed by the CH2 group of MLY159 and the carboxyl oxygen OE2 of Glu156 is shown in Figure 1C. The length of the cohesive interaction is $3.27 \AA$. Forty new intra molecular (NZ)CH...O contacts in the range 3.2 to $4.0 \AA$ A were generated because of the methylation, of which 25 interactions were between 3.2 to $3.8 \AA$ (see Additional file 1). The large number of cohesive intra molecular contacts generated helps immobilize the flexible regions of the protein molecules (Figure 1D), which is crucial for the formation of stable intermolecular contacts and may lower the entropic cost of crystallization. The temperature factor of the MLY159NZ was 12.18. The addition of methyl groups to the amine nitrogen and the for- mation of cohesive bonds through the protonated carbon of the methyl group seemed to have significantly lowered the B factor of the NZ atom of MLY159 indicating a localized side chain. Glu216 forms two strong (NZ)CH...O cohesive bonds with the methyl groups of MLY159 (Figure 2A). The B factors of the OE1 and OE2 atoms of Glu216 involved in the formation of the hydrogen bond were 14.3 and 12.36 respectively. The low B factors demonstrate immobilization of the Glu216 side chain possibly because of the new (NZ)CH...O bonds. Similarly, the B factors of both the carboxyl oxygens of Glu156 participating in (NZ)CH...O interactions were also low. B factors of 16.64 for the OE1 oxygen and 20.24 for the OE2 oxygen indicate the side chain of Glu156 to be severely restricted in movement. The $\mathrm{B}$ factors, however, need to be interpreted cautiously in absence of a structure of the unmodified protein for comparison.

\section{Generation of inter molecular contacts}

The methyl carbons of all the dimethylated lysine residues were involved in the formation of multiple new symmetry-generated intermolecular contacts. The synthesis of the intermolecular contacts was initiated by gradual evaporation of the solvent in presence of different chemicals. Commercially available sparse matrix screens under oil were used for screening the best chemical environment for formation of intermolecular bonds $[19,20]$. The structure reveals 96 new symmetry-generated inter molecular contacts in the range $3.2-5.0 \AA$ involving the (NZ)CH group and 28 of these inter molecular contacts are of the (NZ)CH...O type and in the range 3.2 - $4.0 \AA$ (see Additional file 2). A significant number of these interactions were within the optimal range of $3.2-3.7 \AA$ for $\mathrm{CH}$...O hydrogen bonds [21]. The intermolecular contacts involving the methyl groups of MLY112 and MLY201 are shown in Figures 2B and 2C respectively. The B factors for the NZ atoms of MLY112 and MLY201 were 25 and 15 respectively. The new intra and inter molecular contacts formed by the covalently linked methyl groups with the surrounding residues seem to have lowered the $\mathrm{B}$ factor values of the NZ atom indicating a localized side chain. This helps the packaging of the molecules in the crystal and the formation of a compact crystalline lattice.

Although the contacts formed were predominantly (NZ)CH...O bonds, a few (NZ)CH...N bonds were also formed (Figure $3 \mathrm{~A}$ and $\mathrm{3B}$ ). The side chain nitrogens of Arg35 interact with the CH1 methyl carbon of MLY172. Water molecules surrounding these interactions possibly help disperse the excess positive charge on the nitrogens by bridging ionic interactions [22]. The NH1 nitrogen of Arg35 forms a hydrogen bond with water 119, which is hydrogen bonded to the carboxylic oxygens, OD1 and OD2, of Asp 39. Similarly, the NH2 nitrogen of Arg35 forms a hydrogen bond with water 117 , which is hydro- 

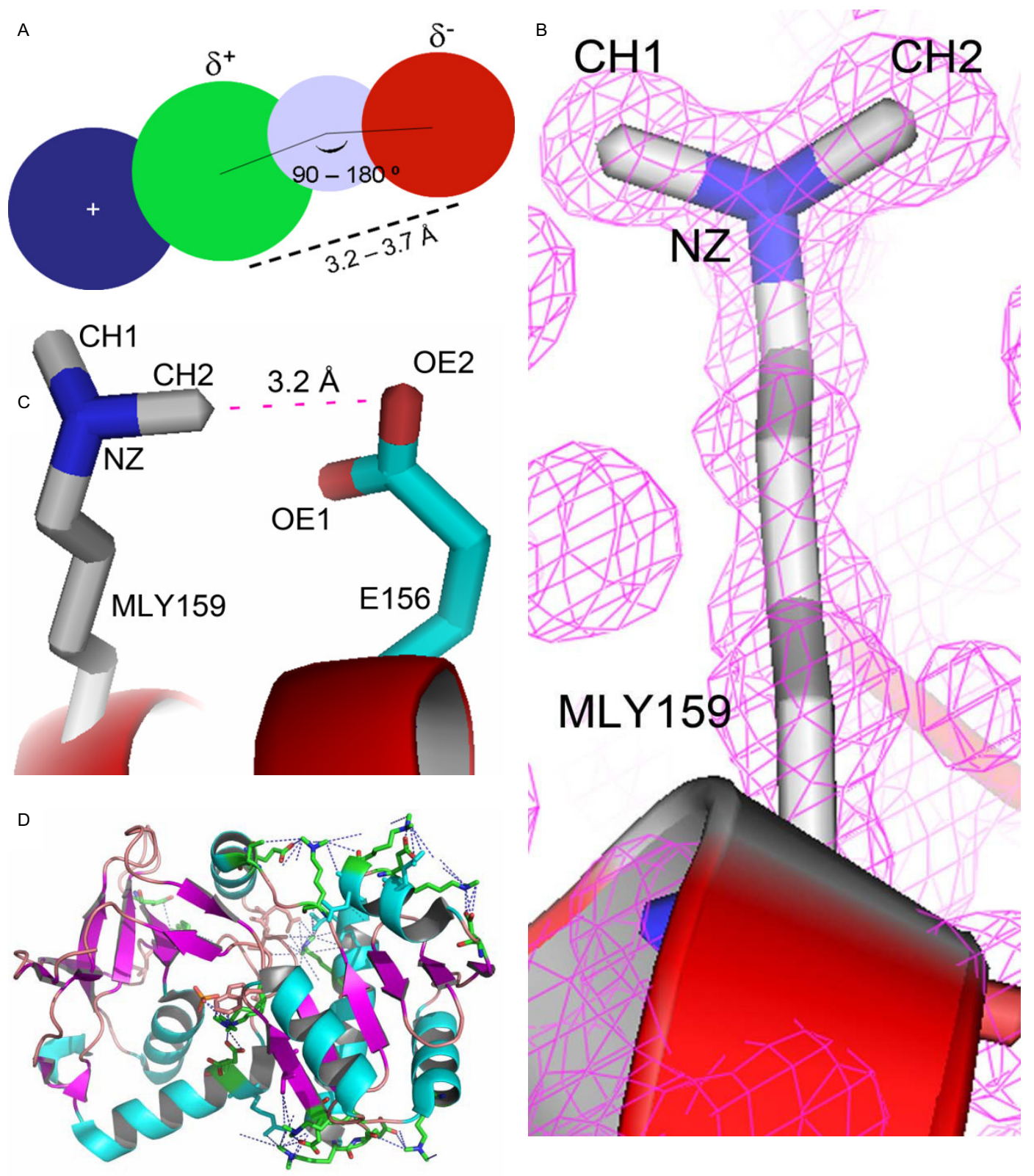

\section{Figure I}

Nature of (NZ)CH...O contacts of the methylated nuclease. A. Diagrammatic representation of the (NZ) CH...O bond. The side chain amine nitrogen (NZ) of lysine residues (blue circle) polarizes the covalently linked methyl carbon (green circle). The polarized methyl carbon acts like a proton donor and forms ionic interactions with neighbouring carboxyl oxygens (red circle). The optimal range for the (NZ)CH...O bond distance is between $3.2-3.7 \AA$. The angle of the approach of a proton towards the lone pair of electrons is generally between $90-180^{\circ}$. In order to calculate the angle, the position of hydrogen (grey circle) for X-ray structures is usually deduced. B. Electron density for the dimethylated lysine MLYI59. The $2 \mid$ Fo |- | Fc | electron density map was contoured at 1.5 б. C. The protonated methyl carbon, CH2, of MLYI59 is seen forming a $3.6 \AA$ (NZ) $\mathrm{CH}$... O bond with the carboxyl oxygen of EI56. The amino acids are represented as sticks. The (NZ)CH...O bond is shown as a dashed magenta line. D. A cartoon representation of the modified protein showing the dimethylated lysines engaged in the formation of numerous intra molecular (NZ) CH... $\mathrm{O}$ contacts. Such contacts localize side chains and loops in space, resulting in a compact protein molecule. (NZ)CH...O bonds are shown as dashed blue lines. 


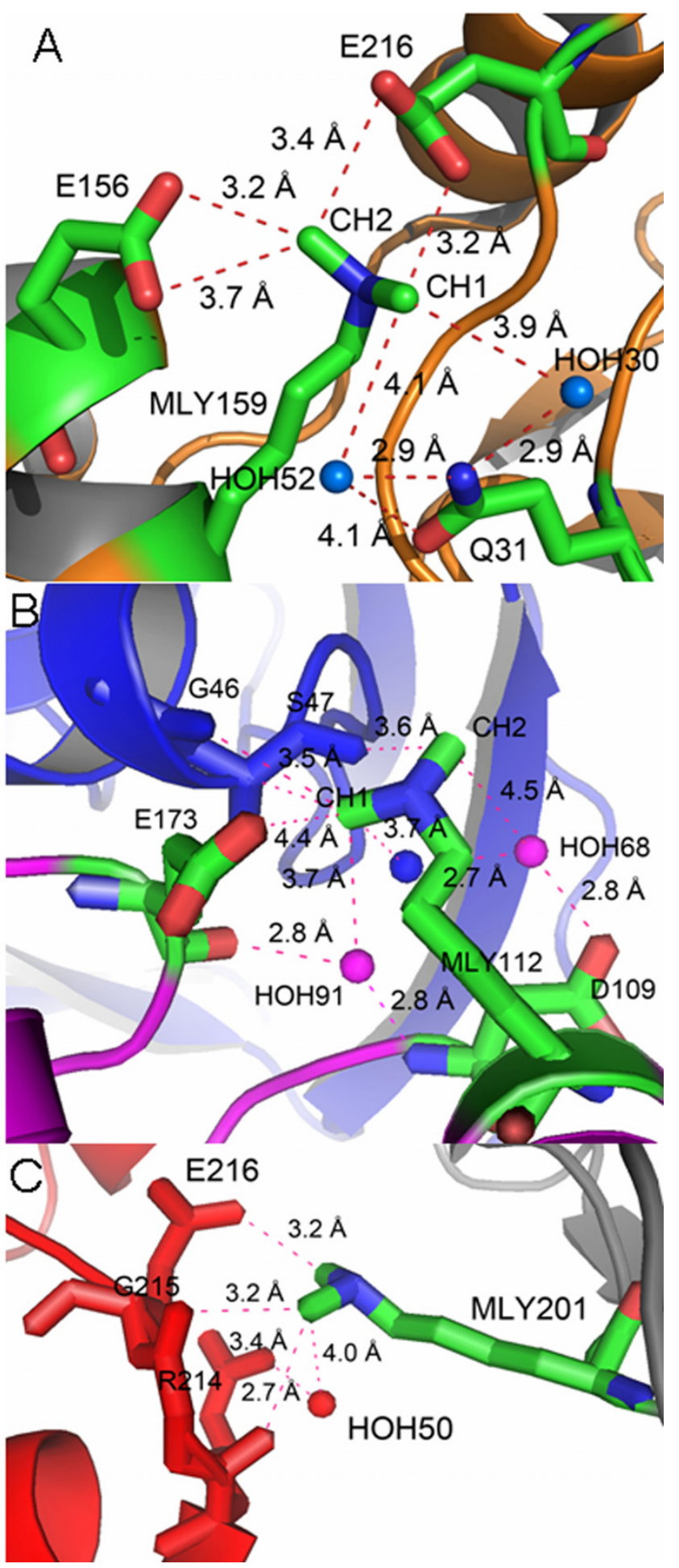

Figure 2

Generation of intra and inter molecular (NZ)CH...O contacts for the nuclease. A. The intra molecular (NZ)CHO contacts generated for MLYI59 as a result of the chemical modification of the nuclease are shown as dashed magenta lines. B, C. The symmetry generated inter molecular contacts synthesized for MLY I I 2 and MLY20I during the crystallization of the nuclease are shown as magenta dashed lines. Residues involved in the formation of $(\mathrm{NZ}) \mathrm{CH}$...O bonds are represented as sticks while water molecules are represented as spheres. 
gen bonded to the carbonyl oxygen of $G \ln 170$. The excess positive charge on the Arg35 nitrogens is relayed to the carboxyl oxygens of Asp35 and carbonyl oxygen of Gln170 via water molecules (data not shown). Similarly, in case of $G \ln 31$, the excess positive charge is relayed to the side chain OE1 oxygen atom.

In addition, a significant number of water molecules were observed to form (NZ)CH... O contacts (Figure 3C).

\section{Analytical ultracentrifugation}

The $26 \mathrm{kD}$ protein was subjected to analytical ultracentrifugation analysis in order to determine the effect of the reductive methylation protocol on the purity, aggregation state and shape of the protein. The sedimentation velocity experiment results revealed that both non-methylated and methylated proteins were pure, homogenous and monomeric (Figure 4). However, a qualitative decrease in the diffusion co-efficient (D) could be observed for the methylated protein (Figure 4B). This is consistent with decreased flexibility of side chains and a more compact structure in the methylated protein.

\section{Thermodynamic stability of nuclease before and after modification}

In order to further investigate the mechanism by which chemical modification of the protein affected the ease of crystallization, we performed equilibrium denaturation experiments using heat or the chaotropic agent guanidinium chloride $(\mathrm{GdmCl})$ as denaturants. The nuclease contains three Trp residues, of which two (W74 and W102) are buried in the folded structure, making intrinsic fluorescence a sensitive probe of global structural changes. We also used far-UV circular dichroism (CD), which monitors changes in secondary structure. Both proteins were found to be extremely thermostable and no secondary structural changes were detected below $90^{\circ} \mathrm{C}$ (not shown). However, we found that the proteins could be completely unfolded in $4 \mathrm{M} \mathrm{GdmCl}$ at $25^{\circ} \mathrm{C}$ (Figure 5A). Further, thermal denaturation could be achieved in the presence of a non-denaturing concentration of $\mathrm{GdmCl}$ (Figure 5B). Interestingly, we found that the chemical modification resulted in a decrease in the mid-point of unfolding in both $\mathrm{GdmCl}$ and thermal denaturation (Figures 5A, B), which corresponds to a decrease in the free energy of unfolding, $\Delta G_{\mathrm{U}, \mathrm{H} 20}$, of $18 \pm 3 \mathrm{~kJ} / \mathrm{mol}$ (Table 1). Surface lysines are likely to be involved in salt-bridges or other favourable interactions that may be lost on methylation, resulting in a decrease in stability. Another explanation is that there is a decrease in entropy in the folded state of the modified protein due to formation of intra molecular (NZ)CH...O interactions involving the introduced methyl groups, which reduces the flexibility of the protein. Solvent effects may also contribute to the observed difference in stability, for example, greater ordering of water mole- cules around hydrophobic methyl groups at the protein surface could also lead to a greater loss in entropy on folding for the methylated protein. We also observed that the $\lambda_{\max }$ of the fluorescence spectrum for the native state was blue-shifted for the modified protein, whereas the $\lambda_{\text {max }}$ for the denatured states was similar (Figure 5A). This is consistent with a more compact structure and better solvent exclusion of buried Trp residues in the folded state of the chemically modified protein. Reduced entropy of the solvated folded structure, together with the formation of additional favourable interactions in the crystalline state, may account for the greater ease of crystallization after the chemical modification

\section{Discussion}

Structure determination holds the key to unravelling the mechanisms by which proteins drive the machinery of all living organisms for survival and propagation. In spite of several dramatic technological advances in automation and information science, the key step for successful crystallographic structure determination - production of high quality crystals - continues to remain a resource intensive bottleneck [1]. A first step towards addressing this bottleneck would be to identify the variables involved in crystallization. The nature of the protein is the single most important factor that influences crystallization. From a crystallization point of view - size, charge, hydrophobicity, hydrophilicity, flexibility, oxidation state and posttranslational modifications like phosphorylation, glycosylation and myristylation - define the nature of a protein. Since the nature of the protein often varies with function, there is no universal crystallization strategy that would work for all proteins. To maximize the chances of crystallization it is crucial to identify and target an attribute of the protein that would have a profound effect on the crystallization. Surface lysines offer one such target. Lysines almost always reside on the surface of proteins. The solvent exposed side chains of lysines are highly mobile and prevent the formation of inter molecular contacts essential for the assembly of a crystalline lattice [18]. Defects observed in protein crystals like low resolution and twinning is also a manifestation of the flexibility of the side chains found on the surface of the protein.

Locking the side chain amine nitrogen of lysines with the electron negative carboxyl oxygens of glutamic and aspartic acid side chains via cohesive ionic interactions will result in the immobilization of these side chains (Figure 2). Although the presence of $\mathrm{CH}$...O bonds has been demonstrated in proteins, nucleic acids and carbohydrates [21-27], the (NZ)CH...O bonds introduced in the current study have never been described before. Methyl groups are very effective in mediating and bridging the physical distances between the free amine nitrogen and the side chain carboxyl oxygens for the formation of cohesive ionic inter- 


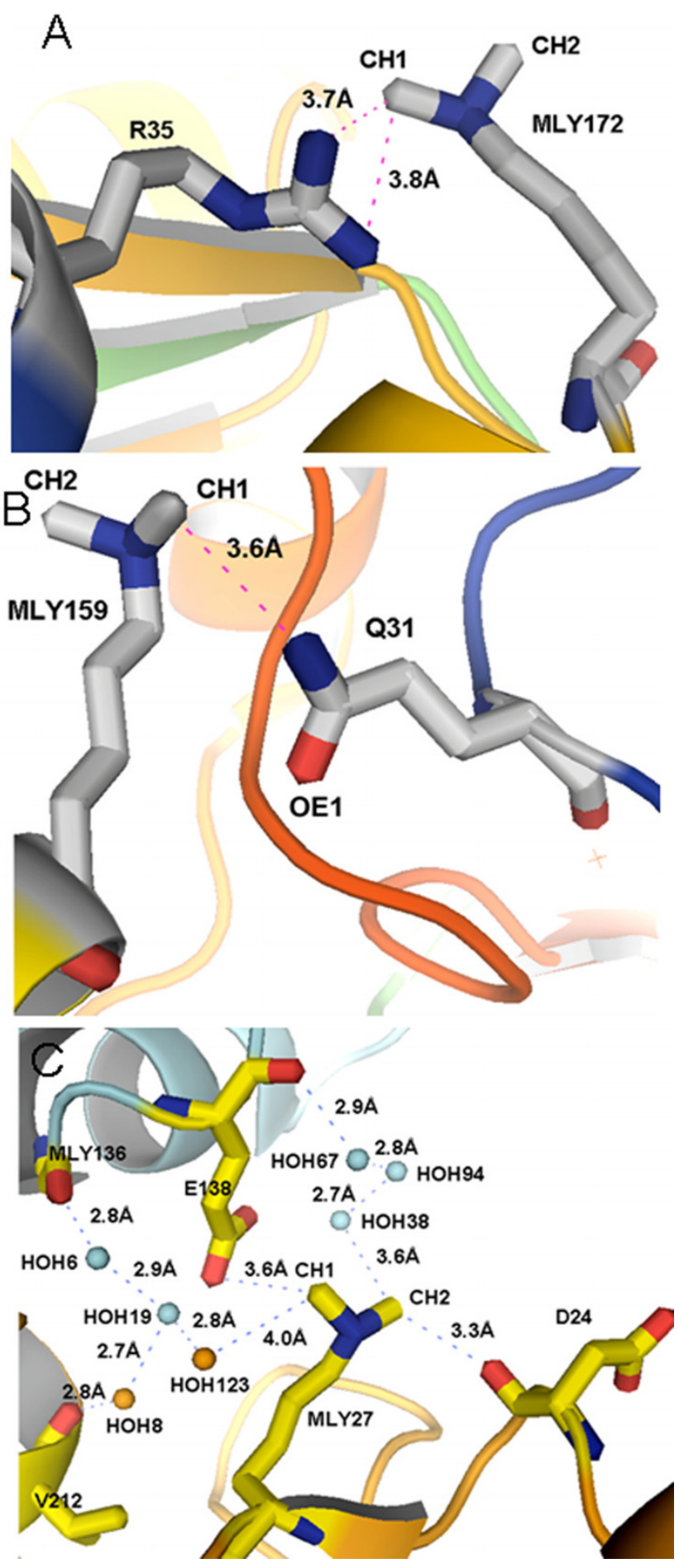

Figure 3

(NZ)CHN contacts and water molecules in (NZ)CHO bonds observed for the methylated nuclease. A, B. The side chain nitrogens of R35 and Q3I are seen forming (NZ)CHN bonds with the methyl groups of MLYI72 and MLYI59 respectively. Excess positive charge on the nitrogen is dispersed via ionic interactions mediated by water molecules [22] or as in case of Q3 I, relayed directly to electron negative oxygen OEI. C. A number of water molecules are seen participating in the formation of (NZ) CHO bonds. Residues involved in the formation of (NZ)CHN and (NZ)CH...O bonds are represented as sticks while water molecules are represented as spheres. 


\section{A}
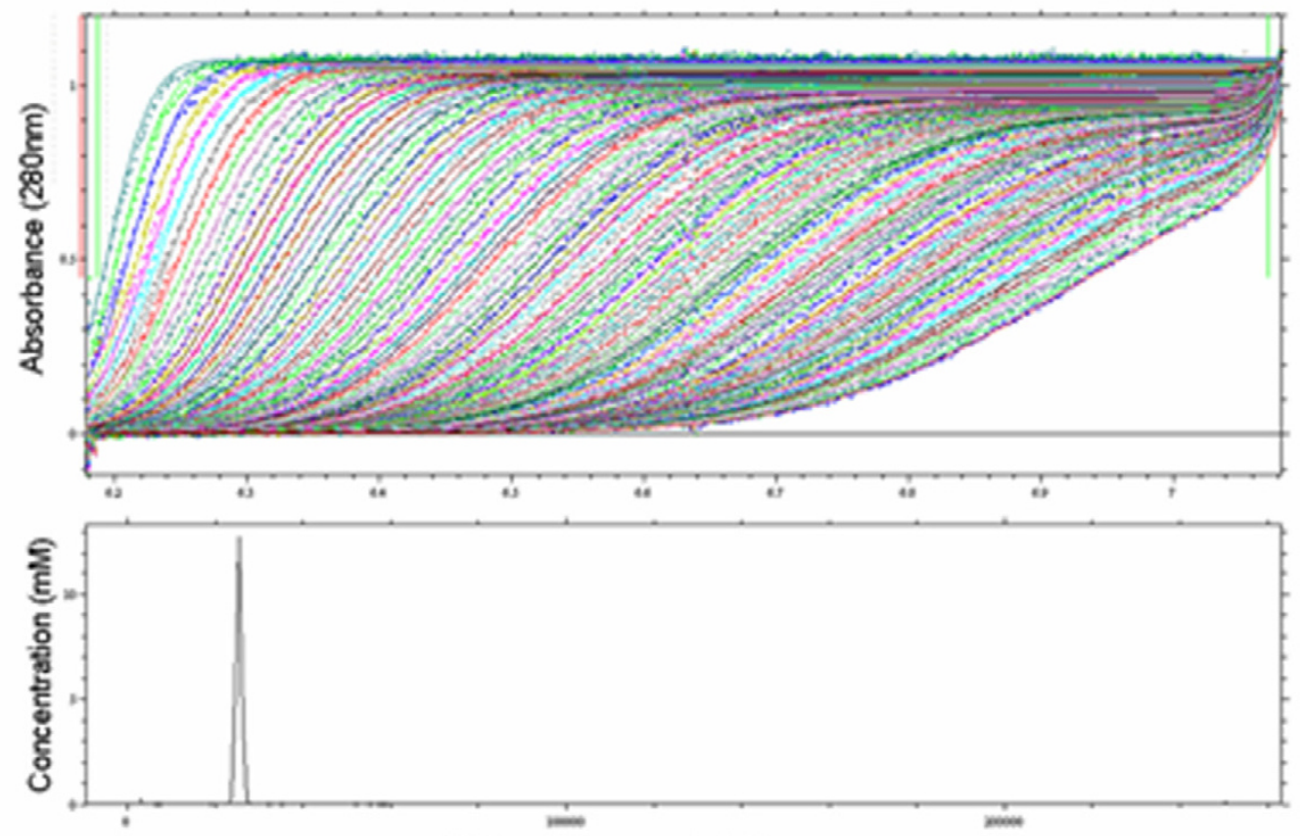

Molecular mass ( daltons)
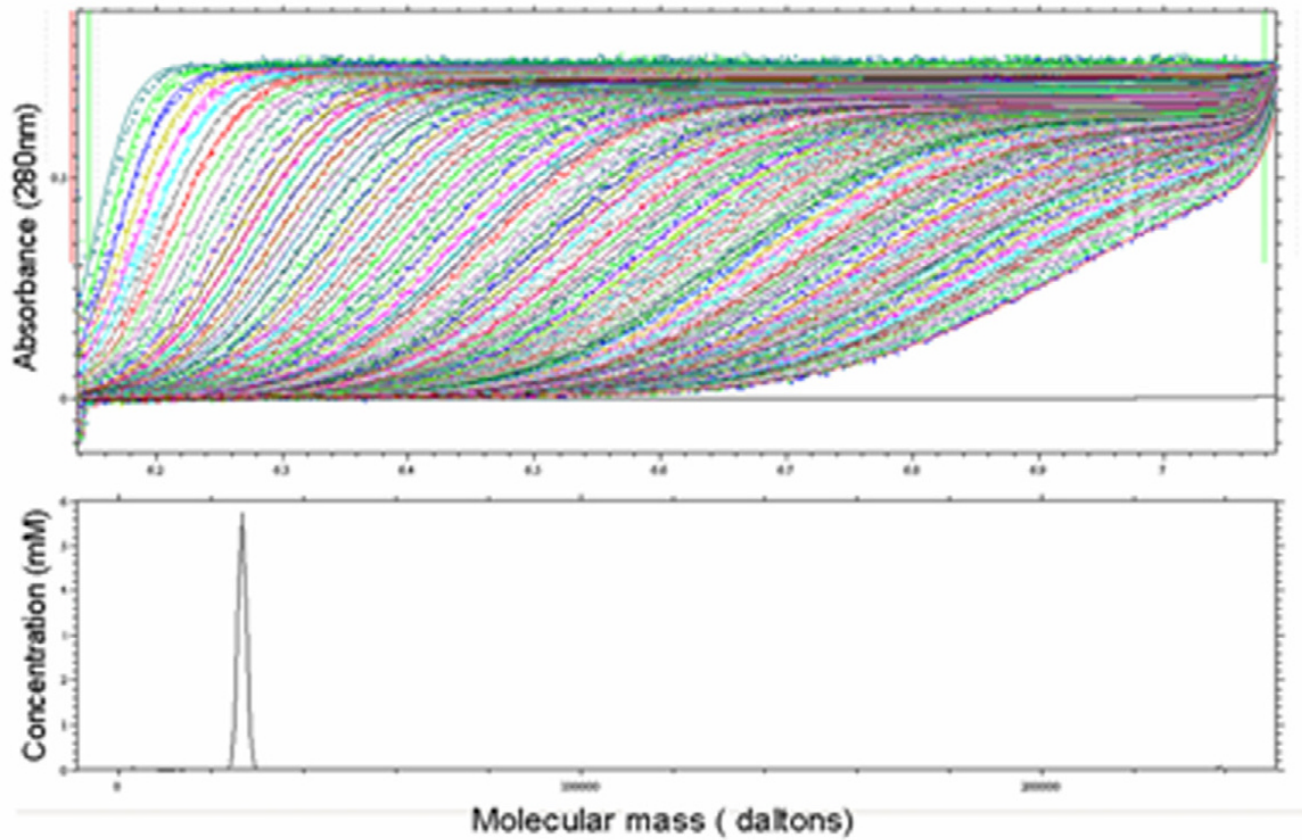

Figure 4

Analytical ultracentrifugation analysis of methylated and non-methylated protein. The methylated (A) and nonmethylated (B) protein were subjected to analytical ultracentrifugation analysis as described in Methods. The sedimentation velocity experiments showed the protein remained pure and monomeric after the chemical modification. There was an obvious decrease in the diffusion co-efficient for the protein after the methylation indicating a more compact shape. 
A

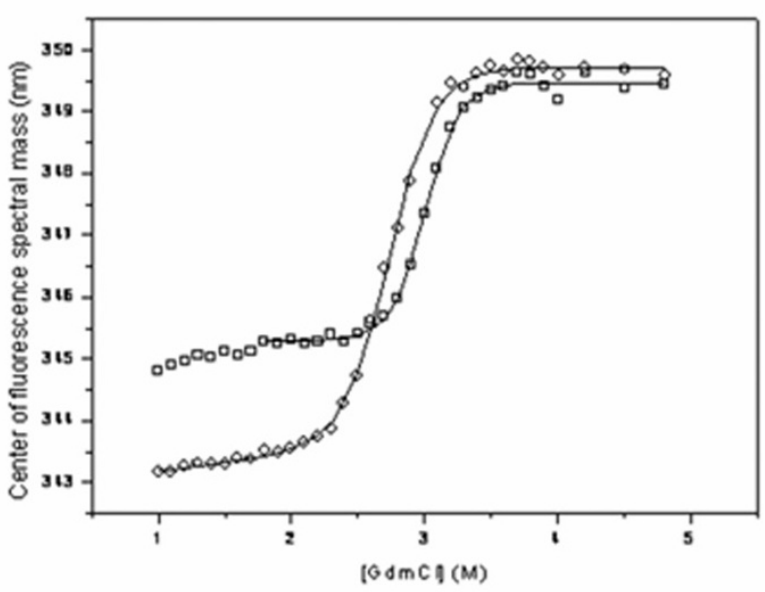

$\mathrm{B}$

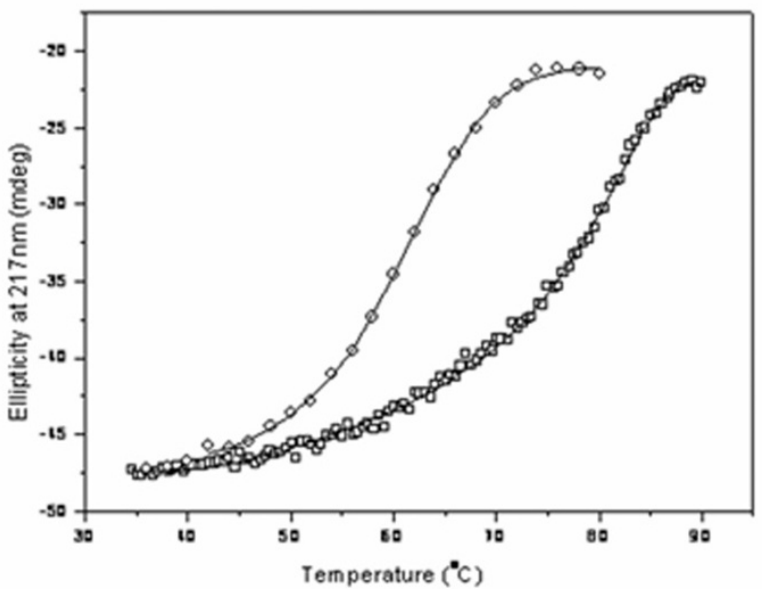

Figure 5

Comparison of thermodynamic stability of modified and non-modified protein. A. GdmCl-induced equilibrium denaturation of the nuclease (squares) and its modified variant (circles) in $20 \mathrm{mM}$ phosphate buffer $\mathrm{pH} 7.4$ at $25^{\circ} \mathrm{C}$. The protein concentration was I $\mu \mathrm{M}$. The fit to a two-state model is shown and the parameters obtained are displayed in Table I. B. Thermally-induced equilibrium denaturation of the nuclease (squares) and its modified variant (circles) in $20 \mathrm{mM}$ phosphate buffer $\mathrm{pH} 7.4$ in the presence of $2.5 \mathrm{M} \mathrm{GdmCl}$. The protein concentration was $25 \mu \mathrm{M}$. The fit to a two-state model is shown and the free energy value obtained after extrapolation to standard conditions $\left(25^{\circ} \mathrm{C}\right.$ in the absence of $\left.\mathrm{GdmCl}\right)$ is the same within error as that obtained by $\mathrm{GdmCl}$ denaturation (Table I).

actions. Methyl groups can be covalently linked to the free amine nitrogen (NZ) with very high specificity using formaldehyde as the methyl group donor in the presence of dimethylamine borane complex [3]. The amine nitrogen sitting adjacent to the methyl carbon, (NZ)CH, has an inductive effect on the methyl carbon resulting in a highly polarized carbon, which can act as a proton donor. The negative charge required for the electro neutrality is compensated by the lone pair of electrons of carboxyl oxygens found on the side chains of neighbouring glutamic and aspartic acid residues. The net effect is a cohesive ionic
(NZ)CH...O interaction between the side chains leading to a compact, rigid protein molecule with localized side chains and loops. The positive charge on the methyl carbon can also be dispersed indirectly via the participation of water molecules (Figure 3C).

The maximum and minimum distances for a cohesive $\mathrm{CH}$... O bond have been set at $3.2 \AA$ and $3.7 \AA$ A respectively, depending on the direction of the approach of the proton towards the lone pair of electrons of oxygen [21]. Deviations in the above distances are frequently observed

Table I: Comparison of thermodynamic parameters for ParB before and after modification§

\begin{tabular}{llll}
\hline Protein & $\begin{array}{l}{[\mathrm{GdmCl}]_{1 / 2}} \\
(\mathrm{M})\end{array}$ & $\begin{array}{l}\mathrm{m} \\
(\mathrm{kJ} / \mathrm{mol} / \mathrm{M})\end{array}$ & $\begin{array}{l}\Delta \mathrm{G}_{\mathrm{U}, \mathrm{H} 2 \mathrm{O}} \\
(\mathrm{kJ} / \mathrm{mol})\end{array}$ \\
\hline Nuclease & $3.01 \pm 0.01$ & $19 \pm 1$ & $57.4 \pm 3.0$ \\
Nuclease (modified) & $2.74 \pm 0.01$ & $14.4 \pm 0.5$ & $39.4 \pm 1.4$ \\
\hline
\end{tabular}

$\S$ The values for $\mathrm{GdmCl}$ denaturation were obtained by fitting data to a standard 2-state model [29] where [GdmCl] $]_{1 / 2}$ is the midpoint and $m$ the slope of the denaturation transition. $\Delta G_{U, \mathrm{H} 2 \mathrm{O}}$ is the free energy of unfolding at $25^{\circ} \mathrm{C}$ in the absence of denaturant. The errors shown are the standard errors from the fit. The dependence of $\Delta G_{U}$ on the $\mathrm{GdmCl}$ concentration is given by $\Delta G_{(\mathrm{GdmCl})}=\Delta \mathrm{G}_{\mathrm{u}}-\mathrm{m}[\mathrm{GdmCl}$. Conditions were as described in the legend for Figure 5A. The thermal denaturation data (Figure 5B) was also fitted to a 2-state model and was extrapolation to standard conditions $\left(25^{\circ} \mathrm{C}\right.$ in the absence of denaturant) as described previously [30]. The value of $\Delta G_{U, H 2 O}$ thus obtained is the same within the error as that shown above. 
owing to the steric interactions between the atoms involved in the formation of the (NZ)CH...O bonds and the surrounding residues. In the present study, except for a couple of contacts involving MLY221, the minimum distance of all the $\mathrm{CH}$...O bonds was $3.2 \AA$. The only short contacts - a $3.0 \AA$ A link between the CH2 methyl carbon of MLY221 and a phosphate oxygen, and a $3.1 \AA$ bond between the carboxyl oxygens of E36 and the MLY221 methyl carbons, resulted in the distortion of the conformation of the MLY221 and the glutamic acid (Figure 6), suggesting steric clashes due to the excessive closeness of the methyl carbon and the oxygen atoms. Thus, $3.2 \AA$ seems to be the minimum van der Waals limit for all (NZ)CH...O interactions. Detailed analysis of the electron density map showed no other obvious changes to any other amino acid confirming the specificity of the modification.

The methylated protein lost its ability to cleave DNA (results not shown). The dimethylation of K221 sitting at the edge of the active site may sterically hinder access of the active site to the incoming DNA. It is also possible that an overall conformational change in the protein induced by the chemical modification affects the catalytic site and compromises the function of the protein. However, such loss-of-function due to methylation has not been reported previously. Further studies are warranted in order to determine the exact cause for the inactivation of the methylated protein.

When a protein is set up for crystallization, the molecules are moving randomly in search of compatible bonding partners. Some of the inter molecular contacts generated during the course of random collisions are sustained. As more and more solvent evaporates, a number of these interactions become permanent. Eventually it leads to one of two possible outcomes. If the inter molecular bonding is heterogeneous, as in case of protein molecules that assume more than one conformation due to the presence of unstructured domains, flexible loops and side chains, or presence of partially unfolded regions, this will result in a disordered protein aggregate commonly referred to as precipitate. Poorly diffracting or defective crystals are also a consequence of the conformational flexibility of protein molecules. A homogenous inter molecular bonding pattern between protein molecules, as in the case of structurally rigid molecules, results in optimal packing of the molecules in a crystal lattice. A direct manifestation of the chemical modification of the protein is the reduction in number of degrees of freedom available to the protein for assuming different conformations. Introduction of (NZ)CH...O bonds curbs the movement of side chains and fixes their orientation in space. This produces uniform bonding partners and decreases the steric clashes between molecules during the packing of the lattice. The

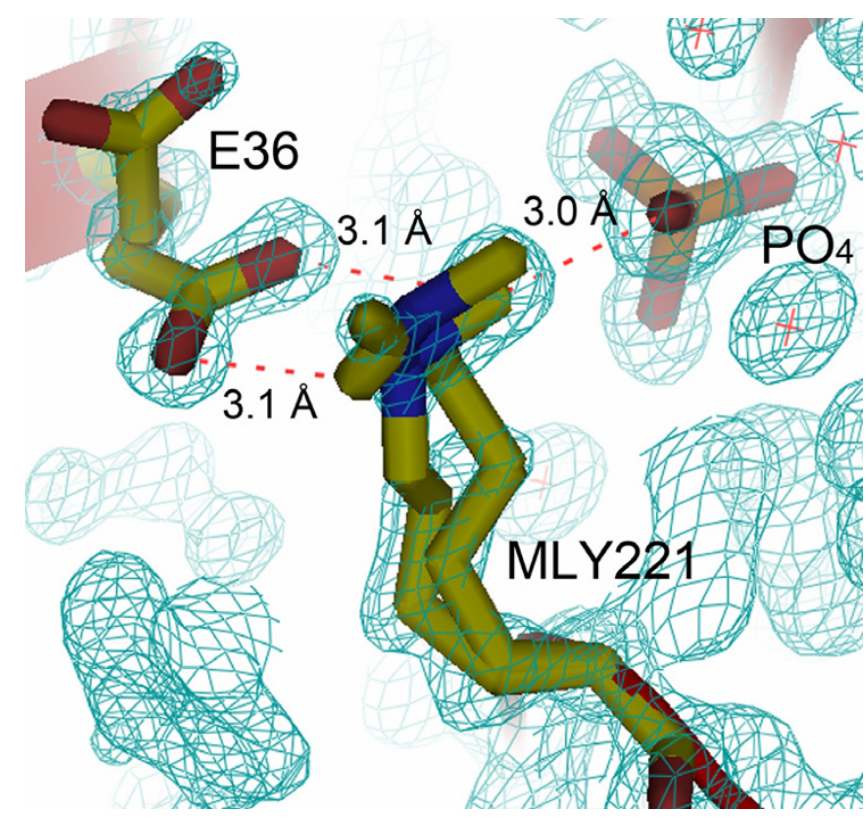

Figure 6

Occupancy of MLY22I. MLY22I shows 2 conformations. Conformation A has occupancy of $65 \%$. The distance of the oxygen atoms of E36 and the $\mathrm{PO}^{-}{ }_{4}$ ligand from the (NZ)CH group of the MLY22I is shorter than the permissible limit of $3.2 \AA$ for (NZ)CHO bonds, resulting in the distorting of MLY22I and E36. The 2 | Fo | - | Fc | electron density map was contoured at $\mathrm{I} .5 \sigma$

observed reduction in the free energy of folding for the modified protein is consistent with reduced flexibility leading to lower entropy in the native state; ordering of water molecules around the surface-exposed methyl groups may also contribute to this. Crystallization will be favoured for the modified protein by the reduction in entropy of the solvated structure and the involvement of the methyl groups in inter molecular (NZ)CH...O interactions upon crystallization

A pre-requisite to the success of the crystallization strategy described here is the requirement of a highly pure homogenous protein sample [28] usually obtained by a combination of different chromatography steps (Figure 7). Presence of homologous and heterologous impurities can compromise the effectiveness of the chemical modification of the protein.

\section{Conclusion}

In conclusion, introduction of (NZ)CH...O bonds by reductive methylation of surface lysines as a means to salvage targets is simple, fast, economical and non laborious. It could be the first method of choice for rescuing a target before attempting a more extensive approach involving mutagenesis. 


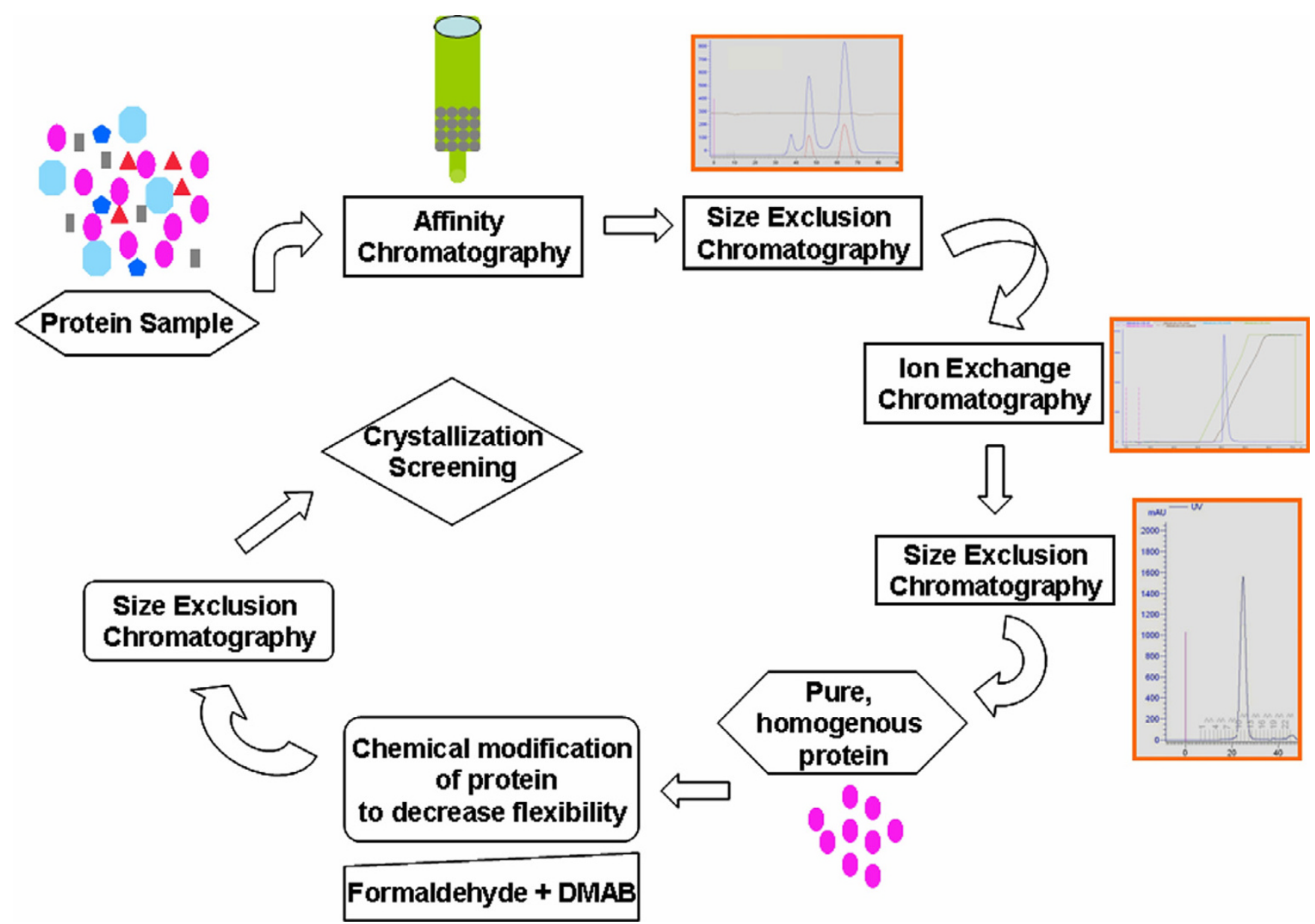

Figure 7

Process flow sheet of the crystallization strategy for the nuclease. The protein sample is purified to homogeneity using a combination of chromatographic methods. The pure and homogenous protein is chemically modified in order to localize the side chains and loops. The resultant compact protein molecule is then screened against a variety of chemical environments using commercially available sparse matrix screens to determine the best condition for the self-assembly of the protein molecules into a crystalline lattice.

\section{Methods}

\section{Protein production and purification}

The $26 \mathrm{kD}$ ParB nuclease was expressed with a N-terminal hexa Histidine tag. The gene was PCR amplified from the genomic DNA of Pyrococcus furiosus and cloned into pET28 a vector (Invitrogen). E coli BL21 cells containing the plasmid were grown in LB cultures. The protein was purified using affinity chromatography followed by size exclusion chromatography. Nucleic acids were removed by hydroxyapatite chromatography (GE Healthcare). Further purification was achieved by an ion exchange step. The protein was exchanged into crystallization buffer $(20 \mathrm{mM}$ Tris, pH8.0, $200 \mathrm{mM} \mathrm{NaCl}$ ) using a size exclusion column.

\section{Chemical modification}

Methylation of the protein was done as described before [3] using formaldehyde and dimethylamine-borane complex (DMAB). In brief, $10 \mathrm{mg} / \mathrm{ml}$ of protein in a $1.5 \mathrm{ml}$ eppendorf tube covered with aluminium foil was mixed with $40 \mu \mathrm{l}$ of $1 \mathrm{M}$ solution of formaldehyde (Sigma) and $20 \mu \mathrm{l}$ of $1 \mathrm{M}$ solution of DMAB (Sigma) in the dark at $4^{\circ} \mathrm{C}$. The reaction mixture was incubated under shaking conditions for $2 \mathrm{~h}$, after which the chemical additions were repeated. Finally, $10 \mu \mathrm{l}$ of DMAB was added and the reaction mixture was incubated overnight. Excess chemicals were removed by size exclusion chromatography.

\section{Crystallization}

Methylated and non-methylated protein was set up for crystallization under oil as described before $[19,20]$. 1- $\mu$ l crystallization drops contained $0.5 \mu \mathrm{l}$ protein mixed with 
$0.5 \mu \mathrm{l}$ of crystallization solution. Commercially available sparse matrix screens (Hampton Research, Molecular Dimensions) were used for crystallization screening. Crystals for structure determination were produced using a precipitant solution consisting of $600 \mathrm{mM}$ sodium dihydrogen phosphate, $2.4 \mathrm{M}$ di-potassium hydrogen phosphate, $200 \mathrm{mM}$ sodium chloride, $100 \mathrm{mM}$ HEPES, p H 7.3.

\section{Data collection and structure determination}

Data collection and structure determination will be described elsewhere.

\section{Analytical Ultracentrifugation}

Analytical sedimentation velocity experiments were carried out using a ProteomeLab ${ }^{\mathrm{TM}}$ XL-I protein characterization system (Beckman Coulter). An-60Ti rotor was used to centrifuge a $10 \mathrm{mg} / \mathrm{ml}$ protein sample suspended in 50 $\mathrm{mM}$ phosphate buffer, $150 \mathrm{mM} \mathrm{NaCl}, \mathrm{pH}$ 7.2, at 60,000 $\mathrm{rpm}$. Absorbance was read at $280 \mathrm{~nm}$. A set of 93 scans were collected at $1 \mathrm{~min}$ intervals. Data was analyzed using Sedfit software

\section{Equilibrium denaturation experiments}

All fluorescence denaturation experiments were performed in $20 \mathrm{mM}$ phosphate buffer $\mathrm{pH} 7.4$ at $25^{\circ} \mathrm{C}$, with a final protein concentration of $1.2 \mu \mathrm{M}$. Samples of modified and unmodified protein were mixed with different concentrations of $\mathrm{GdmCl}$ and allowed to equilibrate overnight before measurements were taken. Refolding experiments were also performed, by denaturing the protein for $8 \mathrm{~h}$ in $6 \mathrm{M} \mathrm{GdmCl}$, then diluting the protein to give different final concentrations of $\mathrm{GdmCl}$ as for unfolding experiments. The intrinsic fluorescence spectra were recorded between 300 and $400 \mathrm{~nm}$ after excitation at $280 \mathrm{~nm}$ in a Hitachi F-4500 spectrofluorimeter. The fluorescence data were plotted as the centre of spectral mass as described previously [29]. GdmCl denaturation was found to be reversible and the data were fitted to a 2 -state model [30].

Far-UV CD experiments were performed on a Pi-star 180 instrument (Applied Photophysics, UK) using a cell of 1 mm optical path length and the same buffer as for fluorescence experiments. The protein concentration was $25 \mu \mathrm{M}$. The temperature was changed at a rate of $1^{\circ} \mathrm{C}$ per $10 \mathrm{~min}$, with a step size of $0.5^{\circ} \mathrm{C}$, for both heating and cooling. Thermal denaturation was found to be reversible and the data were analyzed as described previously [31]

\section{List of abbreviations used}

MLY - Methylated lysine

(NZ) - Side chain amine nitrogen

\section{Authors' contributions}

NS, JC, and JN produced, purified, and crystallized the protein. NS and CC did the chemical modification and analytical ultracentrifugation analysis. X-YW and SP carried out the thermodynamic characterization of the protein. Z-JL, WT, and JR solved the structure and did the analysis. Z-JL designed the experiments and interpreted the data. B-CW and ZR conceived the study and participated in its design and co-ordination. NS, SP, and Z-JL drafted the manuscript. All authors read and approved the final manuscript.

\section{Accession numbers}

Coordinates for the structure reported in the current study have been deposited in the Protein Data Bank under accession code $1 \mathrm{VK} 1$

\section{Additional material}

\section{Additional file 1}

Intra molecular contacts $\$$ of the (NZ)CH group of methylated lysines of the nuclease. The data presented in the table is a list of intra molecular contacts generated during the methylation of the nuclease.

Click here for file

[http://www.biomedcentral.com/content/supplementary/14726807-7-46-S1.doc]

\section{Additional file 2}

Symmetry generated contacts* of the (NZ)CH group of the methylated lysines of the nuclease. The data presented in the table is a list of intra molecular contacts generated during the methylation of the nuclease. Click here for file

[http://www.biomedcentral.com/content/supplementary/14726807-7-46-S2.doc]

\section{Acknowledgements}

This work was funded by the 863 (Grant 2006AA02A316) and 973 (Grants 2006CB91090I, 2006CB910903, 2006CB500703) Projects of the Ministry of Science and Technology of China, the National Natural Science Foundation of China (Grants 30470363, 30670427), the National Institutes of Health (Grant IP50 GM62407), the University of Georgia Research Foundation, the Georgia Research Alliance. Crystallographic data was collected at Southeast Regional Collaborative Access Team (SER-CAT) 22-ID beamline at the Advanced Photon Source, Argonne National Laboratory. Use of the Advanced Photon Source was supported by the U. S. Department of Energy, Office of Science, Office of Basic Energy Sciences, under contract number W-31-109-Eng-38.

\section{References}

I. Stevens R, Wilson I: Industrializing structural biology. Science 200I, 293:5I 9-520.

2. Dale G, Oefner C, D'Arcy A: The protein as a variable in protein crystallization. J Struct Biol 2003, I 42:88-97.

3. Rayment I: Reductive alkylation of lysine residues to alter crystallization properties of proteins. Methods in Enzymology 1997, 276: $171-179$. 
4. Geoghegan K, Cabacungan J, Dixon H, Feeney R: Alternative reducing agents for reductive methylation of amino groups in proteins. Int J Pept Protein Res 198I, 1 7:345-352.

5. Gerken T, Jentoft J, Jentoft N, Dearborn D: Intramolecular interactions of amino groups in I3C reductively methylated hen egg-white lysozyme. J Biol Chem 1982, 257:2894-2900.

6. Means G, Feeney R: Reductive alkylation of amino groups in proteins. Biochemistry 1968, 7:2192-2201.

7. Rypniewski W, Holden H, Rayment I: Structural consequences of reductive methylation of lysine residues in hen egg white lysozyme: An X-ray analysis at I.8Å resolution. Biochemistry 1993, 32:985|-9858.

8. Schubot F, Waugh D: A pivotal role for reductive methylation in the de novo crystallization of a ternary complex composed of Yersinia pestis virulence factors YopN, SycN and YscB. Acto Cryst 2004, D60: $1981-1986$.

9. Kobayashi $M$, Kubota $M$, Matsuura $Y$ : Crystallization and improvement of crystal quality for $\mathbf{X}$-ray diffraction of maltooligosyl trehalose synthase by reductive methylation of lysine residues. Acta Cryst 1999, 55(Pt 4):931-933.

10. Walter T, Meier C, Assenberg R, Au K-F, Ren J, Verma A, Nettleship J, Owens $R$, Stuart $D$, Grimes J: Lysine methylation as a routine rescue strategy for protein crystallization. Structure 2006 14:1617-1622

II. Liu Z-J, Tempel W, Ng J, Lin D, Shah A, Chen L, Horanyi P, Habel J, Kataeva I, Xu H, Yang H, Chang J, Huang L, Chang S-H, Zhou W, Lee D, Praissman J, Zhang H, Newton G, Rose J, Richardson J, Richardson $D$, Wang B-C: The high-throughput protein-to-structure pipeline at SECSG. Acta Crystallogr 2005, 6 I (Pt 6):679-684.

12. Dale G, Kostrewa D, Gsell B, Stieger M, D'Arcy A: Crystal engineering: deletion mutagenesis of the $24 \mathrm{kDa}$ fragment of the DNA gyrase B subunit from Staphylococcus aureus. Acta Crystallogr D Biol Crystallogr 1999, 55: I626-1629.

13. Kwong P, Wyatt R, Robinson J, Sweet R, Sodroski J, Hendrickson W: Structure of an HIV gp I 20 envelope glycoprotein in complex with the CD4 receptor and a neutralizing human antibody. Nature 1998, 393:648-659.

14. Derewenda Z: Rational protein crystallization by mutational surface engineering. Structure 2004, I 2:529-535.

15. Longenecker K, Garrard S, Sheffield P, Derewenda Z: Protein crystallization by rational mutagenesis of surfaceresidues: Lys to Ala mutations promote crystallization of RhoGDI. Acta Crytallogr D Biol Crytallogr 200 I, 57(Pt 5):679-688.

16. Mateja A, Devedjiev Y, Krowarsch D, Longenecker K, Dauter Z, Otlewski J, Derewenda Z: The impact of $\mathbf{G l u} \rightarrow \mathbf{A l a}$ and Glu $\rightarrow$ Asp mutations on the crystallization properties of RhoGDI: the structure of RhoGDI at I.3 $\AA$ resolution. Acta Crytallogr D Biol Crytallogr 2002, 58(Pt | 2): | 983- I99|.

17. Baud $\mathrm{F}, \mathrm{Karlin} \mathrm{S}$ : Measures of residue density in protein structures. Proc Natl Acad Sci USA 1999, 96: 12494-12499.

18. Avbelj F, Fele L: Role of main-chain electrostatics, hydrophobic effect and side-chain conformational entropy in determining the secondary structure of proteins. J Mol Biol 1998 279:665-684.

19. Chayen N, Shaw Stewart P, Maeder D, Blow D: An automated system for micro-batch protein crystallization and screening. Appl Cryst 1990, 23:297-302.

20. D'Arcy A, Elmore C, Stihle M, Johnston J: A novel approach to crystallising proteins under oil. I Crystal Growth 1996, 168: $175-80$

21. Derewenda Z, Lee L, Derewenda U: The occurrence of C-HO hydrogen bonds in proteins. J Mol Biol 1995, 252:248-262.

22. Magalhaes A, Maigret B, Hoflack J, Gomes J, Scheraga H: Contribution of unusual arginine-arginine short-range interactions to stabilization and recognition in proteins. J Protein Chem 1994 13:195-215.

23. Kump $R$, Damewood J: Do nitromethane and molononitrile form C-H...O hydrogen bonds? Implications for molecular recognition by crown ethers. I Chem Soc Chem Commun 1988:62I-622.

24. Taylor R, Kennard O: Crystallographic evidence for the existence of $\mathbf{C}-\mathrm{H}$...O, $\mathbf{C}-\mathrm{H}$...N and $\mathbf{C}-\mathrm{H}$...Cl hydrogen bonds. J Amer Chem Soc 1982, 104:5063-5070.

25. Wahl M, Sundaralingam M: CH...O hydrogen bonding in biology. Trends Biochem Sci 1997, 22:97-102.
26. Desiraju G: The C-H...O hydrogen bond in crystals: What is it? Acc Chem Res 1991, 24:290-296.

27. Donati A, Ristori S, Bonechi C, Panza L, Martini G, Rossi C: Evidences of strong $\mathrm{C}$-HO bond in an o-carboranyl beta-lactoside in solution. J Am Chem Soc 2002, I 24:8778-8779.

28. McPherson A: Introduction to Protein Crystallography. Methods: A Companion to Methods in Enzymology 2004, 34:254-265.

29. Lima L, Zingali R, Foguel D, Monteiro R: New insights into conformational and functional stability of human alpha-thrombin probed by high hydrostatic pressure. Eur J Biochem 2004, 27 I:3580-3587.

30. Maxwell K, Wildes, Zarrine-Afsar A, De Los Rios M, Brown A, Frie C, Hedberg L, Horng J-C, Bona D, Miller E, Vallée-Bélisle A, Main E, Bemporad F, Qiu L, Teilum K, Vu N-D, Edwards A, Ruczinski I, Poulsen F, Kragelund B, Michnick S, Chiti F, Bai Y, Hagen S, Serrano L, Oliveberg M, Raleigh D, Wittung-Stafshede P, Radford S, Jackson S, Sosnick T, Marqusee S, Davidson A, Plaxco K: Protein folding: Defining a "standard" set of experimental conditions and a preliminary kinetic data set of two-state proteins. Protein Science 2005, | 4:602-616.

3I. Su X, Sanbo Q, Xian-Ming P: Thermal and conformational stability of Ssh I Ob protein from archaeon Sulfolobus shibattae. Biochem J 2004, 382:433-440.

32. Potterton $E$, Briggs $P$, Turkenburg $M$, Dodson $E$ : A graphical user interface to the CCP 4 program suite. Acta Cryst 2003, 59(Pt 7): I|3I-I $\mid 37$

Publish with Biomed Central and every scientist can read your work free of charge

"BioMed Central will be the most significant development for disseminating the results of biomedical research in our lifetime. "

Sir Paul Nurse, Cancer Research UK

Your research papers will be:

- available free of charge to the entire biomedical community

- peer reviewed and published immediately upon acceptance

- cited in PubMed and archived on PubMed Central

- yours - you keep the copyright
BioMedcentral 